\title{
A supergiants
}

\section{N. Przybilla ${ }^{1}$, F. Bresolin ${ }^{2}$, K. Butler ${ }^{3}$, A. Kaufer ${ }^{4}$, R.P. Kudritzki ${ }^{2}$ and K.A. Venn ${ }^{5}$}

\author{
${ }^{1}$ Dr. Remeis-Sternwarte Bamberg, Sternwartstrasse 7, D-96049 Bamberg, Germany \\ email: przybilla@sternwarte.uni-erlangen.de \\ ${ }^{2}$ Institute for Astronomy, 2680 Woodlawn Drive, Honolulu, HI 96822, USA \\ ${ }^{3}$ Universitäts-Sternwarte München, Scheinerstrasse 1, D-81679 München, Germany \\ ${ }^{4}$ European Southern Observatory, Alonso de Cordova 3107, Casilla 19001, Santiago 19, Chile \\ ${ }^{5}$ Macalester College, 1600 Grand Avenue, Saint Paul, MN 55105, USA
}

\begin{abstract}
A-type supergiants are the primary targets for the quantitative spectroscopy of individual stars in nearby galaxies because of their intrinsic brightness. An overview is given on the non-LTE techniques required for their analysis. Applications concentrate on placing observational constraints on evolutionary models for massive stars and their host galaxies by detailed abundance analyses. Results from high-resolution studies of A-type supergiants in Local Group galaxies and from intermediate-resolution multi-object spectroscopy of supergiants far beyond the Local Group are summarised.
\end{abstract}

Keywords. Stars: abundances, early-type, evolution, supergiants; galaxies: abundances

\section{Introduction}

Massive stars of $\sim 8-40 M_{\odot}$ cross the A-star regime of the HRD during their post-Main Sequence evolution. Being supergiants at that time they are characterised by extended atmospheres, stellar radii measuring several tens to a few hundred $\mathrm{R}_{\odot}$, and immense luminosities, on the order of $10^{4}$ to several $10^{5} L_{\odot}$. The enormous intrinsic brightness, in coincidence with low Bolometric Corrections, makes BA-type supergiants the primary targets for the young field of extragalactic stellar astronomy. Using 8m-class telescopes these objects become accessible to high-resolution spectroscopy in the galaxies of the Local Group, and to medium-resolution spectroscopy out to distances of several Mpc.

This allows observational constraints to be placed on stellar evolution in a variety of galactic environments, in particular, on the effects of metallicity and rotation on stellar mass loss and the efficiency of chemical mixing. Moreover, important contributions can be made for the study of the galactochemical evolution of the host galaxies through the determination of present-day abundance patterns and gradients. BA-type supergiants can help us to verify classical studies of nebulae and extend the elemental inventory to irongroup and s- \& r-process species. Finally, they can act as extragalactic distance indicators, via application of the wind momentum-luminosity and flux-weighted gravity-luminosity relationships (WLR: Kudritzki \& Puls 2000; FGLR: Kudritzki et al. 2003).

To exploit the full potential, a few complications have to be overcome in model atmosphere analyses. The high luminosities drive stellar winds, to be solved in a hydrodynamical approach, and low atmospheric densities facilitate departures from LTE, which require a simultaneous solution of radiative transfer and statistical equilibrium (e.g., Kubát \& Korčáková 2005, Krtička \& Kubát 2005). In the following, we will concentrate on the latter aspect, as this allows us to draw important conclusions for studies of 'normal' 


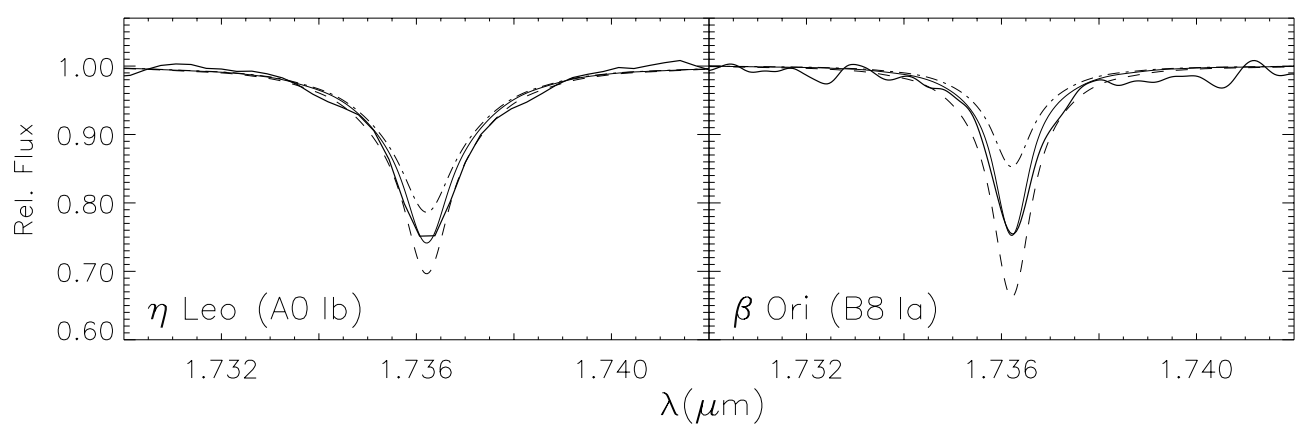

Figure 1. The observed H I $n=4-11$ transition (thick line) in two supergiants is well reproduced by the recommended H i non-LTE model atom of Przybilla \& Butler (2004, thin line), which accounts for accurate electron collision data from quantum-mechanical ab-initio computations for excitation processes. For comparison, synthetic spectra from computations using the Johnson (1972) approximation (dashed) and assuming LTE (dashed-dotted line) are also shown. The computations are performed for stellar parameters derived from the analysis of the visual spectra $\left(\eta\right.$ Leo: $T_{\text {eff }}=9600 \mathrm{~K}, \log g,=2.00 ; \beta$ Ori: $\left.T_{\text {eff }}=12000 \mathrm{~K}, \log g=1.75\right)$. An analogous comparison in Main Sequence stars like Vega indicates that non-LTE departures are underestimated when using the Mihalas et al. (1975) approximation for electron collision rates.

A-stars as well, before discussing recent highlights from the quantitative spectroscopy of extragalactic A-type supergiants.

\section{Quantitative spectroscopy of A-type supergiants}

A-type supergiants were rediscovered as tools for astrophysics in the seminal work of Venn (1995), where modern LTE model atmosphere techniques were shown to suffice for their quantitative analysis using the spectroscopic indicators, ionization equilibria and Stark-broadened hydrogen lines, for the stellar parameter determination (see, e.g., Tanriverdi et al. 2005, Yüce 2005). In the following, the focus shifted from bright, though typically less luminous ( $\mathrm{LC} \mathrm{Ib}$ and Iab) Galactic objects towards supergiants in other galaxies of the Local Group, where only the more luminous stars are accessible to highresolution spectroscopy using the currently available telescopes. Finally, when meeting the challenge of quantitative spectroscopy of supergiants beyond the Local Group only objects near the the Eddington limit are accessible with present-day instrumentation.

The progress on the observational side initiated a reinvestigation of the analyses techniques. Classical line-blanketed LTE atmospheres still turn out to be the best choice for studies of high-luminosity objects at present, however only in combination with massive non-LTE line-formation for the modelling of the photospheric spectrum, i.e., a hybrid non-LTE approach (Przybilla 2002). This is facilitated by the fact that the main atmospheric constituents $(\mathrm{H}, \mathrm{He})$ and the important metal opacities stay close to detailed equilibrium. In the following we discuss the major results from these investigations.

We begin with the most basic element, hydrogen, for which the effects of non-LTE departures in early-type stars were investigated more than three decades ago (e.g., Auer \& Mihalas 1969a,b). Surprisingly, present-day modelling of the hydrogen spectrum fails in reproducing the observed Paschen, Brackett and Pfund lines, both in LTE and non-LTE, though good agreement is obtained for the Balmer lines. All early-type stars are affected, with the discrepancies reaching a maximum in the A-type supergiants, where a mismatch in the line strengths by factors up to 2-3 are found, see Fig. 1 . The spectral features in the visual and IR are consistently reproduced only when commonly-used approximation 


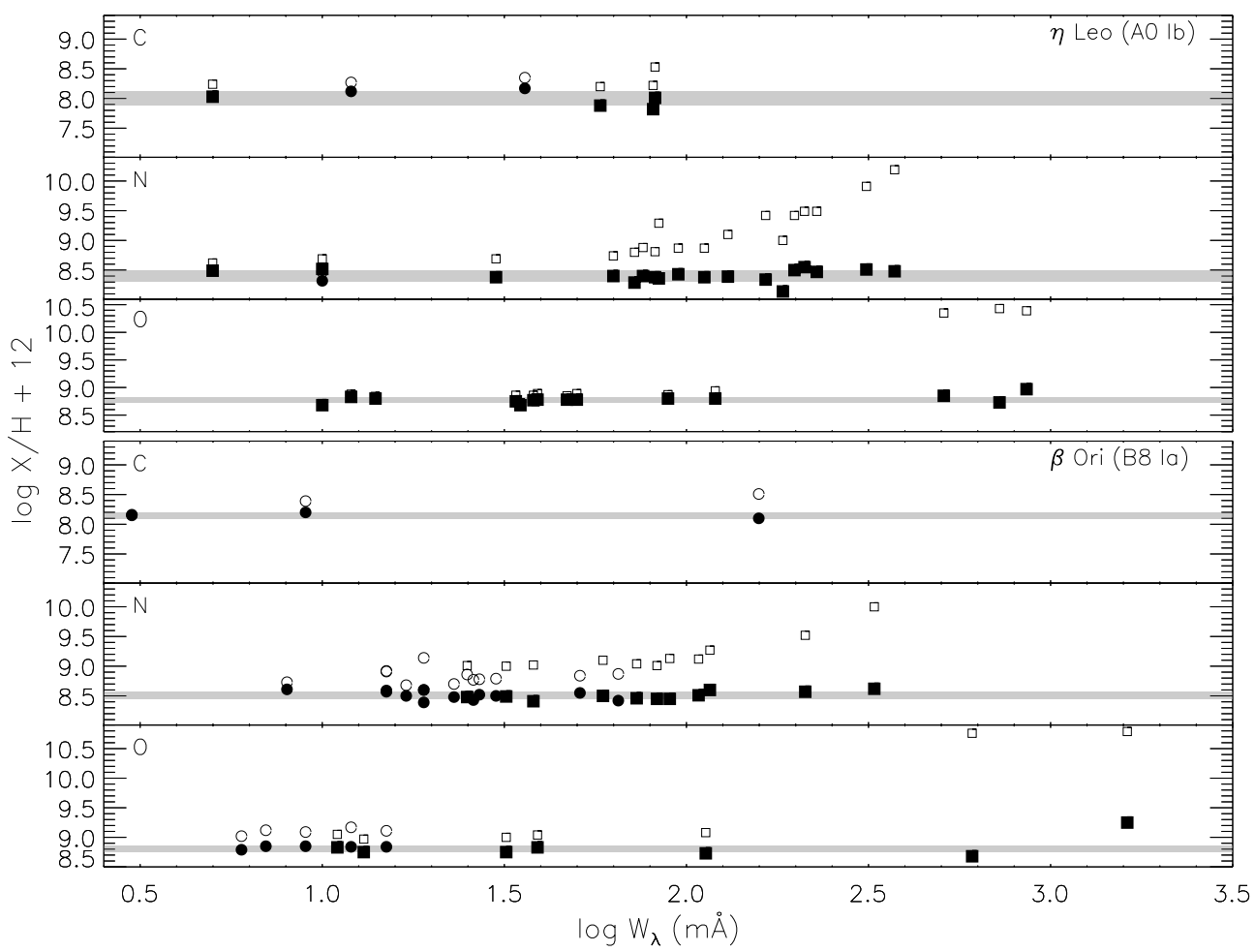

Figure 2. Elemental abundances in two Galactic supergiants from individual spectral lines of CNO plotted as a function of equivalent width: non-LTE (solid) and LTE results (open symbols) for neutral (boxes) and singly-ionized species. The grey bands cover the $1 \sigma$-uncertainty ranges around the mean values. Proper non-LTE calculations reduce the line-to-line scatter and remove systematic trends. Note that even weak lines can show considerable departures from LTE.

formulae for the evaluation of electron-collision excitation rates are dropped in favour of exact data from quantum-mechanical ab-initio computations (Przybilla \& Butler 2004). This is because of a strong sensitivity of the line source-function to non-LTE departures in the Rayleigh-Jeans limit, resulting in an amplification of non-LTE effects in the IR.

Carbon, nitrogen and oxygen are the most abundant metals. All the observed spectral lines in the visual/near-IR originate from high-excitation, (quasi-)metastable levels that favour departures from LTE. Comprehensive non-LTE model atoms, accounting for more sophisticated atomic data than previously possible (Przybilla et al. 2000, 2001, Przybilla \& Butler 2001) allow us to reproduce the entire observed CNO spectra to an unprecedented degree of accuracy, see Fig. 2. In particular, non-LTE abundance analyses remove systematic trends that trouble the LTE approach, and help to reduce the statistical uncertainties from the line-to-line scatter of typically $\sim 0.2$ dex in the literature down to better than 0.1 dex. Contrary to common assumptions, significant non-LTE abundance corrections by $\sim 0.3$ dex can occur even in the weak line limit.

The next step is to broaden our discussion towards a comprehensive study of the entire spectra of BA-type supergiants. Examples are shown in Fig. 3, where results from non-LTE and LTE abundance analyses of primarily weak lines are compared to the solar standard. From this we conclude that the non-LTE analysis reveals a striking similarity of the Galactic supergiant abundance patterns to the solar abundance distribution. This is also found for the M31 object, at slightly higher average metallicity. Fewer chemical 


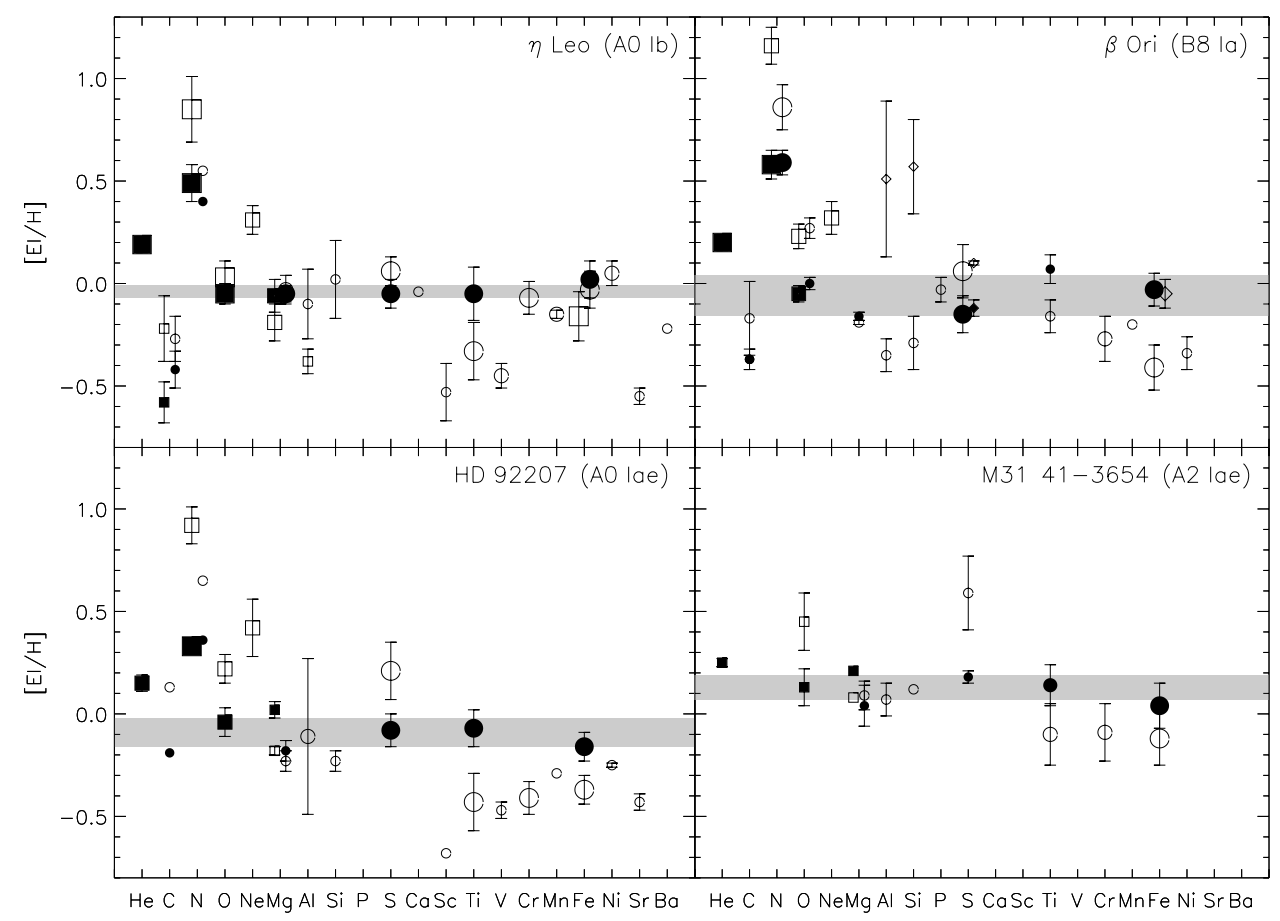

Figure 3. Abundance patterns for four Galactic and M31 supergiants, relative to the solar standard (Grevesse \& Sauval 1998) on a logarithmic scale. Symbol designation as in Fig. 2, with the addition of double-ionized species (diamonds). The symbol size codes the number of spectral lines analysed. Error bars represent $1 \sigma$-uncertainties from the line-to-line scatter and the grey shaded areas mark the deduced stellar metallicity within $1 \sigma$-errors. The non-LTE computations reveal a striking similarity to the solar abundance distribution, except for the light elements which have been affected by mixing with nuclear-processed matter.

species are accessible in this case because of a more restricted spectral coverage and lower $S / N$ of the observations. The light elements $\mathrm{He}, \mathrm{C}$ and $\mathrm{N}$ show marked deviations which are interpreted as mixing of the atmospheric layers with nuclear-processed matter, qualitatively in good agreement with the predictions of the most recent models of massive star evolution (e.g., Maeder \& Meynet 2000). Note in particular that non-LTE calculations can bring several ionization equilibria simultaneously into agreement, thus putting very tight constraints on the stellar parameters. LTE analyses on the other hand produce a large scatter of the individual elemental abundances, and result in increased uncertainties for the different species. They can even suggest $\alpha$-enhancement for the more luminous objects, i.e., apparent overabundances of the $\alpha$-elements, in coincidence with underabundant iron-group elements. This occurs because of selective non-LTE effects, which favour non-LTE line-strengthening in the $\alpha$-elements in analogy to CNO. On the other hand, iron-group elements experience non-LTE line-weakening, because they are characterised by a plethora of energetically-close levels easily coupled via collisions that are collectively subject to non-LTE overionization. Ignoring these non-LTE effects can introduce systematic errors to abundance analyses of more luminous supergiants of typically $\sim 0.3$ dex. Though the astrophysically most important elements are covered by our non-LTE computations, an extension to other chemical species is desirable. However, in many cases a lack of the required atomic data prevents such efforts at present. 


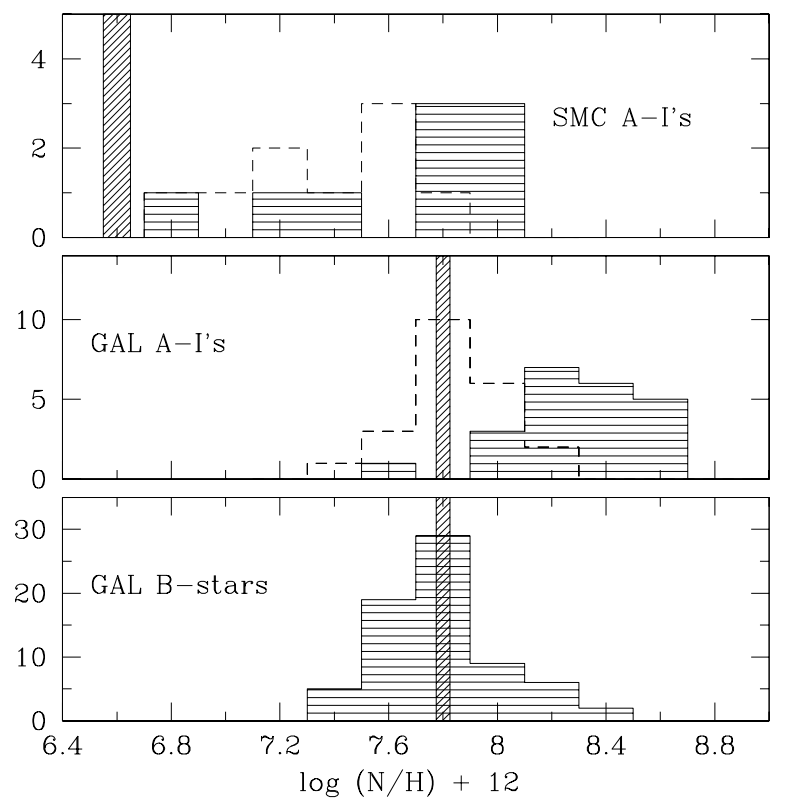

Figure 4. Histogram of nitrogen abundances in Galactic and SMC A-type supergiants, as obtained from an improved non-LTE reanalysis (Venn \& Przybilla 2003) of the Venn (1999, dashed line) data, and of Galactic B-stars (Gies \& Lambert 1992, Cunha \& Lambert 1994), their Main-Sequence progenitors. The initial solar and SMC ISM nitrogen abundances are indicated.

Finally, we like to emphasise that using A-type supergiants as testbeds for the study of non-LTE effects offers a unique opportunity to improve stellar analysis techniques for other classes of stars in general. Due to the universality of atomic properties, sets of reference atomic data should be compiled and verified under the most extreme conditions.

\section{A-type supergiants in the Local Group}

The different galactic environments of the individual Local Group members offer unique opportunities to study the influence of metallicity on the evolution of massive stars. A comparison of Galactic and SMC A-type supergiant abundances (Venn 1999, Venn \& Przybilla 2003) suggests that most of the objects have undergone substantial mixing with CN-cycled material, see Fig. 4. The efficiency of rotational mixing appears to correlate with metallicity since the SMC stars (at $0.2 \times$ solar metallicity) show larger nitrogen enrichments. This is again in good qualitative agreement with the predictions of the latest models of stellar evolution. The effect is a consequence of the reduced metal-line opacity which gives more compact objects and lower mass-loss rates, such that angular momentum losses are considerably reduced, which enables the mixing mechanisms (meridional circulation, shear instabilities) to retain their efficiency. A predicted correlation of mixing efficiency with stellar mass is not verified in this study. Note however, that the sample objects in the SMC are on average more massive than the Galactic supergiants.

The emission spectra of nebulae have been the primary sources for chemical abundances in extragalactic systems beyond the Magellanic Clouds until recently. Despite a widespread use for extragalactic applications, abundance determination techniques for $\mathrm{H}$ II regions (and planetary nebulae) are still subject to a number of inherent problems (see, e.g., Stasińska 2004). Abundances from individual stars in these galaxies open the opportunity to verify such studies with independent and well understood indicators. 


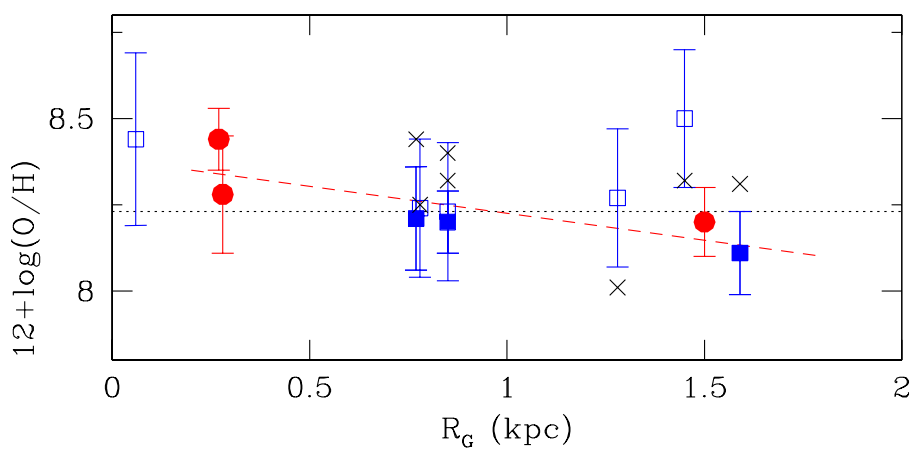

Figure 5. Stellar (Venn et al. 2001, filled circles) and nebular oxygen abundances as a function of galactocentric distance in NGC 6822 suggest a galactic abundance gradient of $\sim-0.1 \mathrm{dex} / \mathrm{kpc}$ (linear least-squares fit: dashed line). H II region abundances are from Pagel et al. (1980, filled squares: abundances derived from $\mathrm{O}$ II and $\mathrm{O}$ III lines, hollow squares: from O II alone). The mean nebular abundance is indicated by the dotted line. Results from a redetermination of the nebular abundances by Pilyugin (2001) are also displayed (St. Andrews crosses). Note that Pilyugin's mean abundance is $\sim 0.1$ dex higher.

Results from a comparison of nebular and stellar oxygen abundances in the dIrr galaxy NGC 6822 are displayed in Fig. 5. Both match within their mutual uncertainties in this low-metallicity case. Unexpectedly comes an indication of an abundance gradient, if a sub-set consisting of the stellar and the more reliable nebular data (i.e., observations showing both, O II and O III) is considered, which, if confirmed by additional measurements, could provide strong constraints on mixing timescales for galactochemical evolution.

While nebulae can provide abundances for a variety of light elements, iron-group and s\& r-process elements are accessible only in stars. These can provide constraints on other important parameters for galaxy evolution, as they trace nucleosynthesis sites complementary to SNeII, which are the main producers of the $\alpha$-elements. Metal-poor dwarf irregular galaxies have attracted particular attention recently, as they can be understood as nearby analogues of the basic building-blocks for hierarchical galaxy formation in the early universe. Spectra of A-type supergiants in the dIrr galaxies SMC, NGC 6822, WLM, Sextans A and GR8 have been obtained with VLT/UVES and Keck/HIRES (Venn 1999, Venn et al. 2001, 2003, Kaufer et al. 2003), requiring exposures of several hours each. At metallicities down to $\sim 0.05 \times$ solar these objects are among the most metal-poor massive stars analysed so far. Mean $\alpha$-element abundance (an average of $\mathrm{O}, \mathrm{Mg}$ and $\mathrm{Si}$ ) to iron abundance ratios for the sample stars as a function of metallicity, $[\mathrm{Fe} / \mathrm{H}]$, are compared to Galactic disk and halo stars of similar metallicity in Fig. 6 . The $[\alpha / \mathrm{Fe}]$ ratios turn out to be roughly solar in these systems, indicating a similar contribution of SNe Ia and II to the chemical evolution as for the young Galactic star population, thus lacking the $\alpha$-enhancement characteristic of old star populations. Globally lower star formation rates than in the solar neighbourhood lead to the lower present-day $[\mathrm{Fe} / \mathrm{H}]$ of the dIrr galaxies.

\section{4. ... and beyond}

The Local Group provides us with a dozen star-forming galaxies, among those three giant spirals, where detailed high-resolution studies of individual luminous stars, primarily BA-type supergiants, are feasible. However, this impressive laboratory is still insufficient for a comprehensive study of galaxy formation and evolution. The step beyond the Local Group has to be taken to investigate all the actively star-forming systems along the Hubble sequence in clusters and other groups of galaxies, and in the field population. 


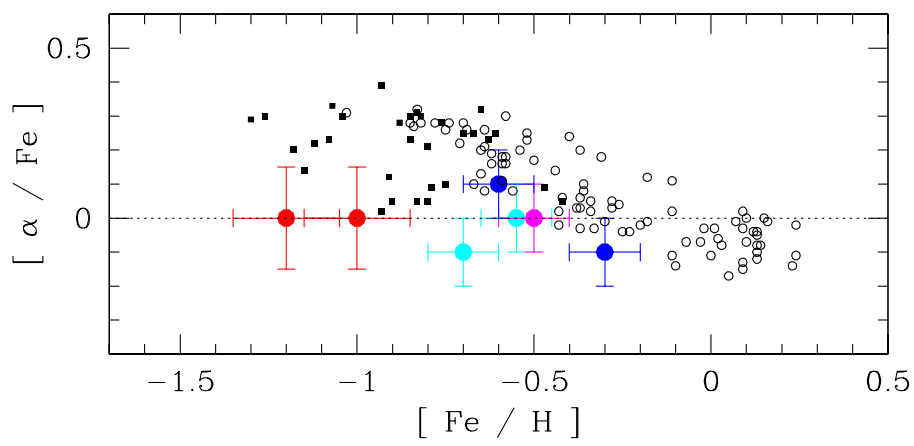

Figure 6. Stellar $[\alpha / \mathrm{Fe}]$ versus $[\mathrm{Fe} / \mathrm{H}]$ in dwarf irregular galaxies (solid circles), from lowest to highest metallicity: Sex A, GR8, WLM, SMC (average), WLM (2nd object), NGC 6822 (average of 3 objects), LMC (average). For comparison, Galactic disk stars (open circles, Edvardsson et al. 1993) and metal-rich halo stars (filled squares, Nissen \& Schuster 1997) are also shown.

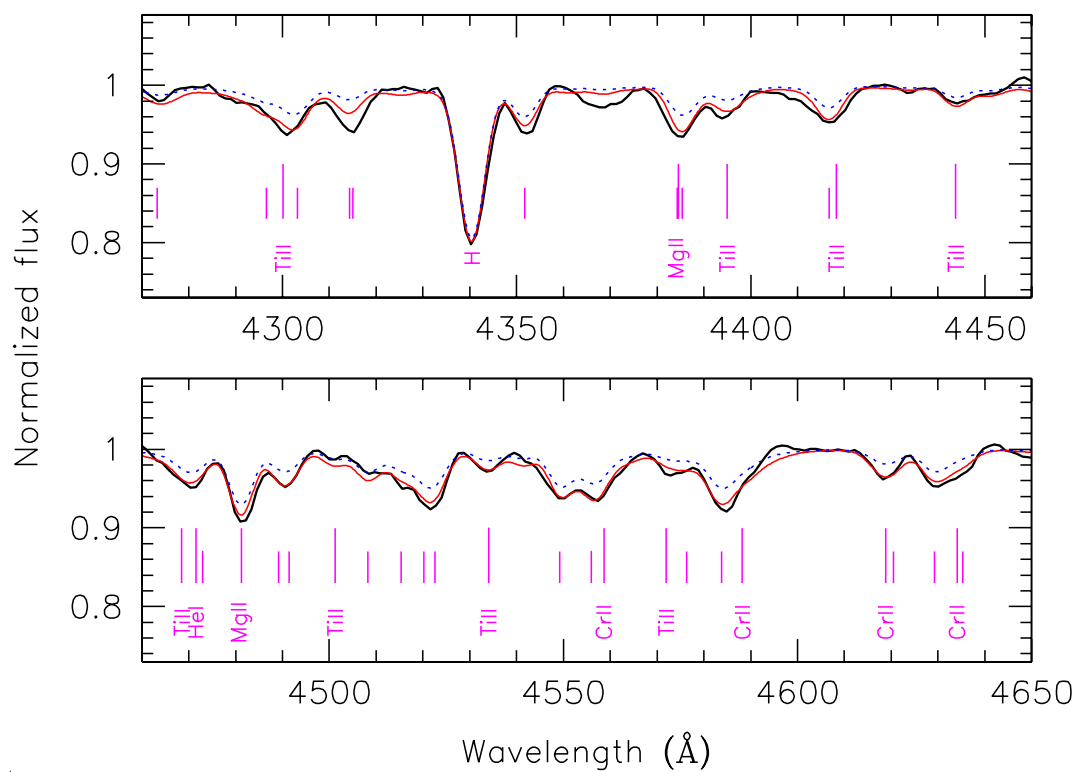

Figure 7. Comparison of the medium-resolution spectrum (thick full line) of a NGC 3621 A-type supergiant $(V=20.47 \mathrm{mag})$ with model predictions $\left(T_{\text {eff }}=9000 \mathrm{~K}, \log g=1.05\right)$ at 0.5 (thin line) and $0.2 \times$ solar metallicity (dotted line). Line identifications are provided (Fe II lines are indicated by the shorter marks). A total of 19 objects in the range $V \approx 20-22$ mag were observed using the multi-object capability of FORS1 on the VLT, of which 10 could be identified as supergiants, requiring a total integration time of $10.7 \mathrm{hr}$.

We have initiated a project to investigate the blue supergiant populations in nearby galaxies beyond the Local Group, using the Fors multi-object spectrograph on the VLT. Quantitative spectroscopy of individual stars out to distances of $\sim 7 \mathrm{Mpc}$ has been performed for the first time, estimating stellar parameters, chemical abundances, reddening, extinction and stellar wind properties. The first target was the field galaxy NGC 3621 (Bresolin et al. 2001). Medium-resolution spectra require spectrum synthesis techniques for the modelling of the entire spectral range to facilitate a closer analysis. While detailed abundance analyses are hampered by line blending, estimates (within roughly $0.2 \mathrm{dex}$ ) are still possible, taking full advantage of the methods presented in Sect. 2, as demonstrated in Fig. 7. The spectrum of the object investigated is well fitted with a chemical 
composition comparable to that of the LMC, i.e., $\sim 0.5 \times$ solar, while a significantly lower metallicity, like that of the SMC can confidently be ruled out.

In the second target, the Sculptor Group galaxy NGC 300, four Fors fields have been observed, yielding spectra of 62 blue supergiants. The first steps to constrain the NGC 300 elemental abundance gradients and the internal reddening have been undertaken (Bresolin et al. 2002, Urbaneja et al. 2003). This will allow us, in addition to the topics already mentioned, to address the influence of two major sources of systematic uncertainty on the Cepheid period-luminosity relation.

\section{References}

Auer, L.H. \& Mihalas, D. 1969a, ApJ 156, 157

Auer, L.H. \& Mihalas, D. 1969b, ApJ 156, 681

Bresolin, F., Kudritzki, R.P., Méndez, R.H., Przybilla, N. 2001, ApJ 548, L159

Bresolin, F., Gieren, W., Kudritzki, R.P., Pietrzyński, G., Przybilla, N. 2002, ApJ 567, 277

Cunha, K. \& Lambert, D.L. 1994, ApJ 426, 170

Edvardsson, B., Andersen, J., Gustafsson, B., et al. 1993, A\&3A 275, 101

Gies, D.R. \& Lambert, D.L. 1992, ApJ 387, 673

Grevesse, N. \& Sauval, A.J. 1998, Space Sci. Rev. 85, 161

Johnson, L.C. 1972, ApJ 174, 227

Kaufer, A., Venn, K.A., Tolstoy, E., Pinte, C., Kudritzki, R.P. 2004, AJ 127, 2723

Kubát, J. \& Korčáková, D. 2005, These Proceedings, 13

Krtička, J. \& Kubát, J. 2005, These Proceedings, 23

Kudritzki, R.P., Bresolin, F., Przybilla, N. 2003, ApJ 582, L83

Kudritzki, R.P. \& Puls, J. 2000, ARA\&A 38, 613

Maeder, A. \& Meynet, G. 2000, ARA\&A 38, 143

Mihalas, D., Heasley, J.N., Auer, L.H. 1975, A Non-LTE Model Stellar Atmosphere Computer Program, NCAR-TN/STR 104

Nissen, P.E. \& Schuster, W.J. 1997, A\&A 326, 751

Pagel, B.E.J., Edmunds, M.G., Smith, G. 1980, MNRAS 193, 219

Pilyugin, L.S. 2001, AESA 374, 412

Przybilla, N. 2002, PhD Thesis, Ludwig-Maximilians-University, Munich

Przybilla, N., Butler, K., Becker, S.R., Kudritzki, R.P., Venn, K.A. 2000, A\&A 359, 1085

Przybilla, N., Butler, K., Kudritzki, R.P. 2001, A\& A 379, 936

Przybilla, N. \& Butler, K. 2001, A\& A 379, 955

Przybilla, N. \& Butler, K. 2004, ApJ 609, 1181

Stasińska, G. 2004, in: C. Esteban et al. (eds.), Cosmochemistry (Cambridge: CUP), p. 115

Tanriiverdi, T., Adelman, S. J., Albayrak, B. 2005, These Proceedings, HP2

Urbaneja, M.A., Herrero, A., Bresolin, F., et al. 2003, ApJ 584, L73

Venn, K.A. 1995, ApJS 99, 659

Venn, K.A. 1999, ApJ 518, 405

Venn, K.A. \& Przybilla, N. 2003, in: C. Charbonnel, D. Schaerer \& G. Meynet (eds.), CNO in the Universe, ASP Conf. Ser. (San Francisco: ASP), vol. 304, p. 20

Venn, K.A., Lennon, D.J., Kaufer, A., et al. 2001, ApJ 547, 765

Venn, K.A., Tolstoy, E., Kaufer, A., et al. 2003, AJ 126, 1326

Yüce, K. 2005, These Proceedings, HP3

\section{Discussion}

Dworetsky: What values did you derive for the microturbulent velocity parameter?

PRZYBILla: The microturbulent velocities apparently correlate with luminosity class. At LC Ib typical values are $\sim 4 \mathrm{~km} \mathrm{~s}^{-1}$, increasing up to $\sim 10 \mathrm{~km} \mathrm{~s}^{-1}$ for objects close to the Eddington limit. The microturbulent velocities remain sub-sonic in all cases. 